\title{
ON THE MOTION OF VISCOUS SHOCKS AND THE SUPERSENSITIVITY OF THEIR STEADY-STATE LIMITS
}

\author{
Jacques G. L. Laforgue and Robert E. O’Malley, Jr. \\ In memory of Charles G. Lange, \\ who knew the true significance of exponential asymptotics.
}

\begin{abstract}
This paper constructs an asymptotic shock-layer solution to the initialboundary-value problem for the partial differential equation

$$
\epsilon\left(f(u) u_{x}\right)_{x}+g(u) u_{x}=u_{t}
$$

in a finite spatial domain with nearly-Dirichlet boundary conditions and, to a much lesser extent, for the reaction-diffusion equation$$
\epsilon^{2}\left(f(u) u_{x}\right)_{x}+h(u)=u_{t} .
$$

Using an exponentially compressed time scale, it provides a differential equation for the shock layer's motion which displays both the slow convergence of the shock to its steady state and the extreme sensitivity of its location to perturbations in coefficients and/or boundary conditions. The key to the analysis is the appropriate handling of asymptotically exponentially small terms.
\end{abstract}

\section{Introduction}

This paper will primarily consider the asymptotic solution of initial-boundary-value problems for the scalar convection-dominated evolution equation

$$
\epsilon\left(f(u) u_{x}\right)_{x}+g(u) u_{x}=u_{t}
$$

where $f$ is positive and smooth, $g$ is smooth, $x$ is one-dimensional, and $\epsilon>0$ is a small parameter. A physically significant and well-studied example is Burgers' equation

$$
\epsilon u_{x x}+u u_{x}=u_{t}
$$

which Bateman (1915) used to describe discontinuous fluid motion by examining the limiting behavior as the nondimensionalized viscosity $\epsilon \rightarrow 0^{+}$(cf. Burgers (1974), Whitham (1974), Kevorkian and Cole (1981), Fletcher (1982), Kreiss and Lorenz (1989), Kevorkian (1990), and Laforgue and O'Malley (1993, 1994) for coverage of more recent work and other applications).

Equation (1) will generally allow solutions which change rapidly in endpoint boundary layer regions, but for appropriate boundary values and hypotheses on $g$, it can instead have an analogous single interior shock layer of $O(\epsilon)$ thickness in space. (See

Received September 21, 1993, revised April 11, 1994.

This work was supported in part by the National Science Foundation under grant number DMS 9107196. 
Garbey (1989) and Garbey and Scroggs (1991) for finite $t$ results.) For convenience, we will introduce

$$
G(u)=\int_{0}^{u} g(s) d s
$$

and rewrite (1) as

$$
\left(\epsilon f(u) u_{x}+G(u)\right)_{x}=u_{t} .
$$

For simplicity, we shall consider the initial-boundary-value problem for (4) on the semi-infinite strip

$$
-1 \leq x \leq 1, \quad t \geq 0
$$

with piecewise-constant data

$$
u=\left\{\begin{array}{cl}
-1 & \text { for } x=-1, t \geq 0 \text { and for }-1 \leq x<x_{0}, t=0 \\
1 & \text { for } x=1, t \geq 0 \text { and for } x_{0}<x \leq 1, t=0
\end{array}\right.
$$

Moreover, we shall assume that $G$ satisfies

$$
\begin{gathered}
G(-1)=G(1) \quad \text { and } G(u)<G( \pm 1) \text { for }-1<u<1, \\
\text { with } g(-1)<0<g(1) .
\end{gathered}
$$

The boundary values \pm 1 provide exact solutions of the integrated steady-state equation and, as $\epsilon \rightarrow 0$, we would expect to obtain a solution $u(x, t, \epsilon)$ of (4)-(6) which increases steeply from -1 to 1 on each constant $t>0$ line in an $O(\epsilon)$-narrow shock layer in the $x$ variable. For definiteness, we take the point $x_{\epsilon}(t)$ where

$$
u\left(x_{\epsilon}(t), t, \epsilon\right)=0
$$

as the shock layer location. Thus, we expect that

$$
u(x, t, \epsilon) \rightarrow \begin{cases}-1, & -1 \leq x<x_{0}(t) \\ 1, & x_{0}(t)<x \leq 1\end{cases}
$$

as $\epsilon \rightarrow 0$, where $x_{\epsilon}(t)$ varies smoothly beginning (nearly) from the prescribed jump (or "zero") location $x_{0}$ of the initial value function. We shall obtain a differential equation for $x_{\epsilon}(t)$ which can be interpreted as arising from an improved RankineHugoniot condition, which takes account of the asymptotically exponentially small errors made by the moving profile in satisfying the boundary conditions. We shall be particularly interested in the long-term evolution of $x_{\epsilon}(t)$ and its ultimate approach to a steady state as $t \rightarrow \infty$.

We note that the asymptotic solution to (4)-(6) for sufficiently large $t$ would feature an endpoint layer near $x=-1$ if the boundary value there were changed to $u(-1, t, \epsilon)=-1+\delta$ for any fixed $\delta>0$ (cf. Figure 1). The layer would appear near $x=1$ if $\delta<0$. This indicates that having an interior shock must be extremely sensitive to perturbations of the boundary values, as was noted computationally by Allen (1988). We will, indeed, find a supersensitivity to asymptotically exponentially small changes in the boundary values $u( \pm 1, t, \epsilon)$ allowing the shock to be displaced by $O(1)$ at $t=\infty$. In Sections 3 and 4 , we provide a detailed study of the dramatic effect of such perturbations. The observed supersensitivity reflects the diminutive size of the dominant eigenvalue of the linearized problem and the resulting need to use an 
asymptotically exponentially long time scale to capture the approach to the steadystate. Our preliminary study of related reaction-diffusion equations in Section 4(b) shows that they, too, are amenable to a parallel attack.

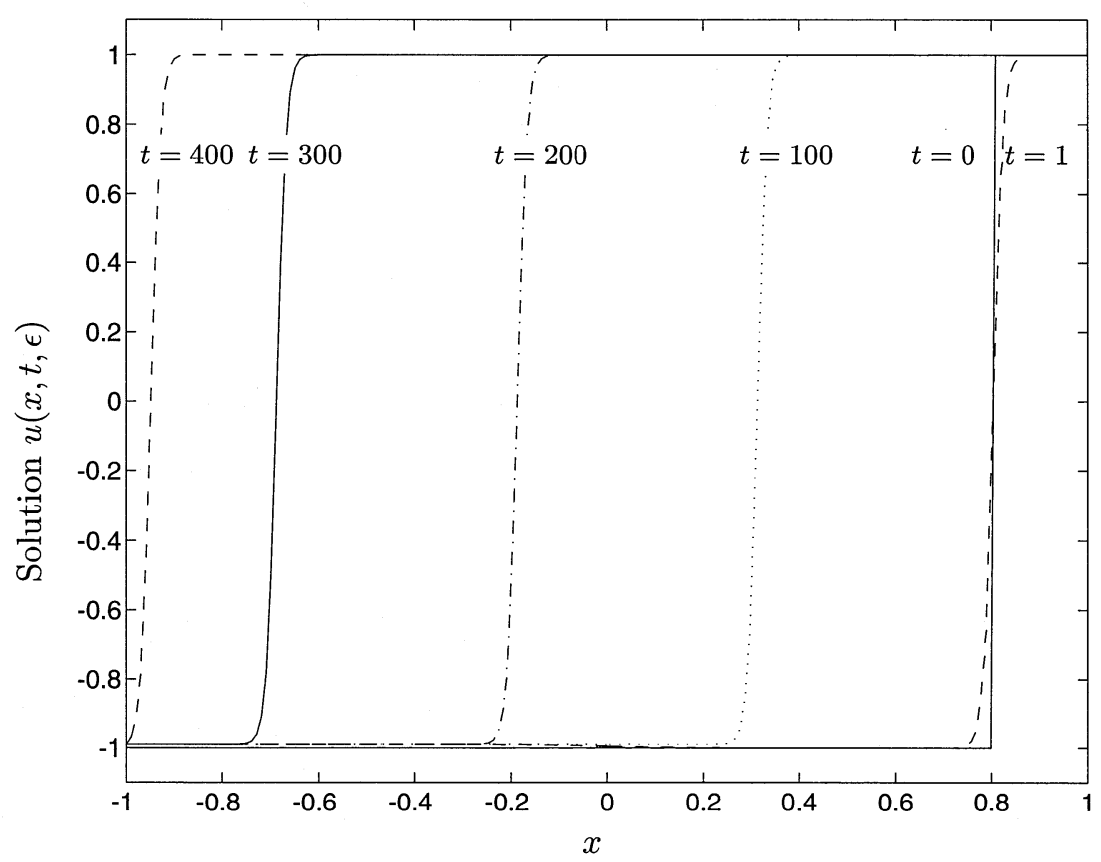

FIgURE 1. Burgers' shock motion for $\epsilon=0.01$ and $u(-1, t)=0.99$.

\section{A preliminary shock layer analysis}

We will first attempt to describe the shock layer for any moderate size $t$ by introducing the stretched spatial coordinate

$$
\eta \equiv\left(x-x_{\epsilon}(t)\right) / \epsilon
$$

which is zero at the shock layer location and has a doubly-infinite interval of values as $\epsilon \rightarrow 0$. Attempting a regular perturbation expansion

$$
u(\eta, t, \epsilon) \sim u_{0}(\eta, t)+\epsilon u_{1}(\eta, t)+\cdots
$$

for the shock-layer solution of (4)-(6), together with a corresponding expansion

$$
x_{\epsilon}(t)=x_{0}(t)+\epsilon x^{1}(t)+\cdots
$$

for the shock-layer location, we naturally need to solve the transformed equation

$$
\left(f(u) u_{\eta}+G(u)\right)_{\eta}=\epsilon u_{t}-\frac{d x_{\epsilon}}{d t} u_{\eta}
$$


on $-\infty<\eta<\infty$, with $u( \pm \infty, t, \epsilon)= \pm 1$ and $u(0, t, \epsilon)=0$. The leading terms of the proposed power series would satisfy the nonlinear ODE problem

$$
\begin{gathered}
\left(f\left(u_{0}\right) u_{0 \eta}+G\left(u_{0}\right)\right)_{\eta}=-\frac{d x_{0}}{d t} u_{0 \eta} \\
u_{0}( \pm \infty, t)= \pm 1, \quad u_{0}(0, t)=0
\end{gathered}
$$

where $t$ is simply a parameter. An integration with respect to $\eta$ from either $\pm \infty$ implies that $f\left(u_{0}\right) u_{0 \eta}+G\left(u_{0}\right)-G( \pm 1)=0$ and $-2 d x_{0} / d t=0$, so

$$
x_{0}(t) \equiv x_{0}
$$

and

$$
u_{0}(\eta, t) \equiv \varphi(\eta) \text { for } \eta \sim \frac{x-x_{0}}{\epsilon}
$$

are independent of $t$. Integrating the translation-invariant equation $f(\varphi) \varphi_{\eta}=G( \pm 1)-$ $G(\varphi)$ by separating variables, we uniquely define the monotonic shock-layer profile $\varphi(\eta)$ implicitly as

$$
\eta=\int_{0}^{\varphi} \frac{f(s) d s}{G( \pm 1)-G(s)}
$$

since we will fix $\varphi(0)=0$ to correspond to the zero of $u(x, t, \epsilon)$. In the special case of Burgers' equation, this yields the familiar shock profile $\varphi(\eta)=\tanh \eta / 2$. In general, the asymptotic behavior of $\varphi$ as $\eta \rightarrow \pm \infty$ is easily determined, since the integral $\eta$ has a logarithmic singularity as $\varphi \rightarrow \pm 1$ (because the integrand has a simple pole at $s= \pm 1)$. Re-expressing (11) as

$$
\begin{aligned}
\eta & =\frac{1}{A_{-}}\left[\ln (\varphi+1)+\int_{0}^{\varphi}\left(\frac{A_{-} f(s)}{G(-1)-G(s)}-\frac{1}{1+s}\right) d s\right] \\
& =\frac{1}{A_{-}}\left[\ln (\varphi+1)-\ln L_{-}\right]+O(\varphi+1) \quad \text { as } \varphi \rightarrow-1,
\end{aligned}
$$

provides us the approximation

$$
\varphi(\eta) \sim-1+L_{-} e^{A_{-} \eta} \quad \text { as } \eta \rightarrow-\infty,
$$

where $L_{-} \equiv \exp \left[\int_{-1}^{0}\left(\frac{A_{-} f(s)}{G(-1)-G(s)}-\frac{1}{1+s}\right) d s\right]>0$ and $A_{-} \equiv-g(-1) / f(-1)>0$. More accurate approximations to $\varphi$ and its derivatives as $\eta \rightarrow-\infty$ also follow readily. Likewise,

$$
\varphi(\eta) \sim 1-L_{+} e^{-A_{+} \eta} \quad \text { as } \eta \rightarrow \infty
$$

where

$$
L_{+} \equiv \exp \left[\int_{0}^{1}\left(\frac{A_{+} f(s)}{G(1)-G(s)}-\frac{1}{1-s}\right) d s\right]>0
$$

and $A_{+} \equiv g(1) / f(1)>0$. The exponential approach of $\varphi(\eta)$ to \pm 1 as $\eta \rightarrow \pm \infty$ could also be shown directly through local linearizations. This results because such motion links fixed points on a heteroclinic orbit (cf. Elphick et al. (1990) and Balmforth et al. (1993) for related work based on analogous homoclinic orbits).

Equating the $O(\epsilon)$ terms in (9) then gives the linear problem

$$
\left(f(\varphi) u_{1}\right)_{\eta \eta}+\left(g(\varphi) u_{1}\right)_{\eta}=-\frac{d x^{1}}{d t} \varphi_{\eta}, \quad u_{1}( \pm \infty, t)=0, \quad u_{1}(0, t)=0 .
$$


Integration yields the unique trivial solution $x^{1}(t)=0$ and $u_{1}(\eta, t)=0$. Continuing to higher orders, we formally obtain the stationary shock solution

$$
u(x, t, \epsilon)=\varphi\left(\frac{x-x_{0}}{\epsilon}\right)+O\left(\epsilon^{N}\right)
$$

for any integer $N>0$, since the higher-order terms all are found to be zero.

We immediately note, however, that (14) cannot be valid at $t=0$ since the initial data has a simple jump at $x_{0}$, while $\varphi$ displays an $O(\epsilon)$-thick transition layer about the point. Anticipating approximate evolution of the solution of (4)-(6) to $\varphi\left(\left(x-x_{0}\right) / \epsilon\right)$ in an initial $O(\epsilon) t$-interval, we naturally introduce the stretched time variable

$$
\tau=t / \epsilon,
$$

together with the corresponding spatial variable $\eta=\left(x-x_{0}\right) / \epsilon$, and seek a solution $u(\eta, \tau)$ of the parameter-free initial-boundary-value problem

$$
\begin{gathered}
\left(f(u) u_{\eta}+G(u)\right)_{\eta}=u_{\tau}, \quad-\infty<\eta<\infty, \tau \geq 0 \\
u(\eta, 0)=\left\{\begin{array}{rrc}
-1, & \eta<0 \\
1, & \eta>0
\end{array} \quad u( \pm \infty, \tau)= \pm 1 .\right.
\end{gathered}
$$

Because (15) isn't generally explicitly solvable, we will use results of Il'in and Oleinik (1964) which show that (for $f(u) \equiv 1$ ) (15) has a unique solution $u(\eta, \tau)$ which tends to its only possible (up to translation) steady state $\varphi(\eta)$, i.e., that

$$
u(\eta, \tau) \rightarrow \varphi\left(\eta-\eta_{0}\right) \quad \text { as } \quad \tau \rightarrow \infty
$$

The phase shift $\eta_{0}$ can be obtained as follows. Integrating the differential equation of (15) from $\eta=-\infty$ to $\infty$ shows that $\int_{-\infty}^{\infty}(u(\eta, \tau)-u(\eta, 0)) d \eta \equiv 0$ for all $\tau$. Letting $\tau \rightarrow \infty$, then, $\eta_{0}$ must satisfy the equal area rule

$$
P\left[\eta_{0}\right] \equiv \int_{-\infty}^{0}\left(\varphi\left(\eta-\eta_{0}\right)+1\right) d \eta+\int_{0}^{\infty}\left(\varphi\left(\eta-\eta_{0}\right)-1\right) d \eta=0 .
$$

In fact, $P^{\prime}\left[\eta_{0}\right] \equiv-2$, so the equation is linear with the unique solution

$$
\eta_{0}=\frac{1}{2}\left[\int_{-\infty}^{0}(\varphi(\eta)+1) d \eta+\int_{0}^{\infty}(\varphi(\eta)-1) d \eta\right] .
$$

As an example, the evolution equation

$$
\epsilon u_{x x}+\frac{1}{5}\left(3 \sqrt{\frac{1+u}{2}}-2\right) u_{x}=u_{t}
$$

has the exact shock profile $\varphi(\eta)=-1+2\left(1+(\sqrt{2}-1) e^{-\eta / 5}\right)^{-2}$ and the phase translation $\eta_{0}=-5(1-\ln (\sqrt{2}+1))$ in the initial $O(\epsilon) t$-layer.

In the special case of Burgers' equation, (15) has the unique solution

$$
u(\eta, \tau)=\frac{e^{\eta / 2} \operatorname{erfc}\left(\frac{-\eta-\tau}{2 \sqrt{\tau}}\right)-e^{-\eta / 2} \operatorname{erfc}\left(\frac{\eta-\tau}{2 \sqrt{\tau}}\right)}{e^{\eta / 2} \operatorname{erfc}\left(\frac{-\eta-\tau}{2 \sqrt{\tau}}\right)+e^{-\eta / 2} \operatorname{erfc}\left(\frac{\eta-\tau}{2 \sqrt{\tau}}\right)}
$$

where $\operatorname{erfc}(z)=\frac{2}{\sqrt{\pi}} \int_{z}^{\infty} e^{-s^{2}} d s$ (cf. Laforgue and O'Malley (1994)). Because $\operatorname{erfc}(-\infty)=2$, we indeed obtain the expected limit $\varphi(\eta)=\tanh \eta / 2$ as $\tau \rightarrow \infty$. The symmetry of $f$ and $G$ imply that of $\varphi$ so $\eta_{0}=0$. For a more general initial 
function $u(x, 0)$ than the jump function of $(5)$, we could often use the method of characteristics to form a shock in finite time and then consider its later evolution as described here (cf. Oleinik (1963), Murray (1968), Lax (1973), and Il'in (1992)).

Ultimately, we must expect the "stationary" shock (14) to migrate from its "initial" location

$$
x_{0}^{\epsilon} \equiv x_{0}+\epsilon \eta_{0}
$$

For Burgers' equation, symmetry shows that the shock must finally move to the midpoint of the spatial interval, $x=0$. In general, any steady state must satisfy the boundary-value problem

$$
\left(\epsilon f(u) u_{x}+G(u)\right)_{x}=0, \quad u( \pm 1, \epsilon)= \pm 1
$$

The two-point problem has the implicit monotonic solution

$$
x=-1+\epsilon \int_{-1}^{u} \frac{f(v) d v}{K(\epsilon)-G(v)}
$$

presuming the constant of integration $K(\epsilon)$ can be selected so that $u(1, \epsilon)=1$. Recalling the assumptions (6) on $G$ and classical techniques for the asymptotic approximation of integrals (cf. Wong (1989) and O'Malley (1991)), it follows that $K(\epsilon)$ is unique and slightly greater than $G( \pm 1)$ and that the asymptotically significant contributions to the integral come only from $v$-values near \pm 1 . To be specific, the conditions $u(1, \epsilon)=1$ and $u\left(x_{\epsilon}(\infty), \epsilon\right)=0$ imply the two asymptotic relations

$$
\frac{2}{\epsilon} \sim-\frac{1}{A_{-}} \ln (K(\epsilon)-G(-1))-\frac{1}{A_{+}} \ln (K(\epsilon)-G(1))
$$

and

$$
\frac{x_{\epsilon}(\infty)+1}{\epsilon} \sim-\frac{1}{A_{-}} \ln (K(\epsilon)-G(-1)) .
$$

Eliminating $\ln (K(\epsilon)-G( \pm 1)) \sim-\left(x_{\epsilon}(\infty)+1\right) A_{-} / \epsilon$ in the first relation yields the limiting steady-state shock location

$$
x_{0}(\infty)=\beta_{0} \equiv \frac{A_{+}-A_{-}}{A_{+}+A_{-}}
$$

This agrees with the known result for Burgers' equation and with results of Howes $(1984,1986)$ for $f(u) \equiv 1$. We note that this steady state is independent of any initial condition $u(x, 0, \epsilon)$ replacing that of (5) and, in particular, of $x_{0}$.

Under hypothesis (6), the classical Rankine-Hugoniot condition (cf. Smoller (1983)) predicts an arbitrary shock location for the inviscid $(\epsilon=0)$ problem. Likewise, the traditional matched asymptotic expansion procedure for singularly perturbed problems fails to specify the steady-state solution (cf. Reyna and Ward (1994a)). Moreover, computations for Burgers' equation show that convergence of the shock layer to its steady state is extremely sluggish (cf., especially, Kreiss and Kreiss (1986)). 


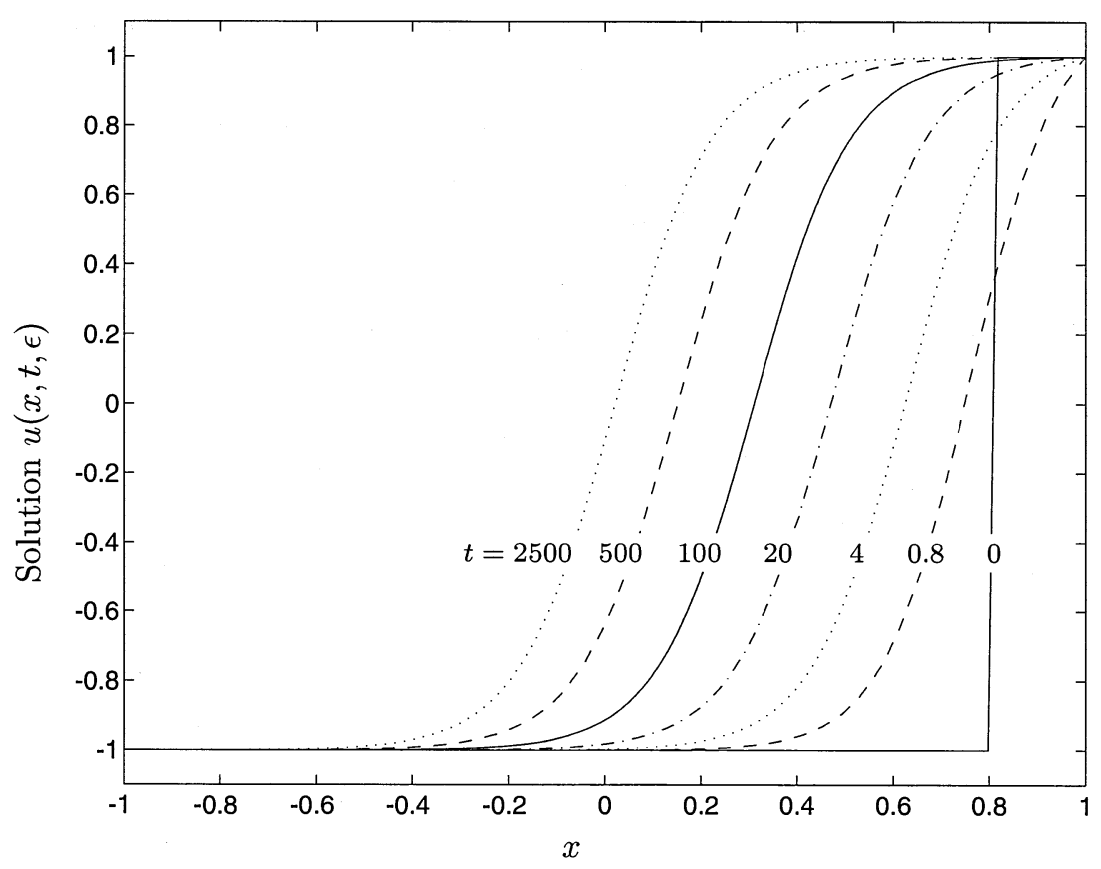

Figure 2. Exponentially slow motion.

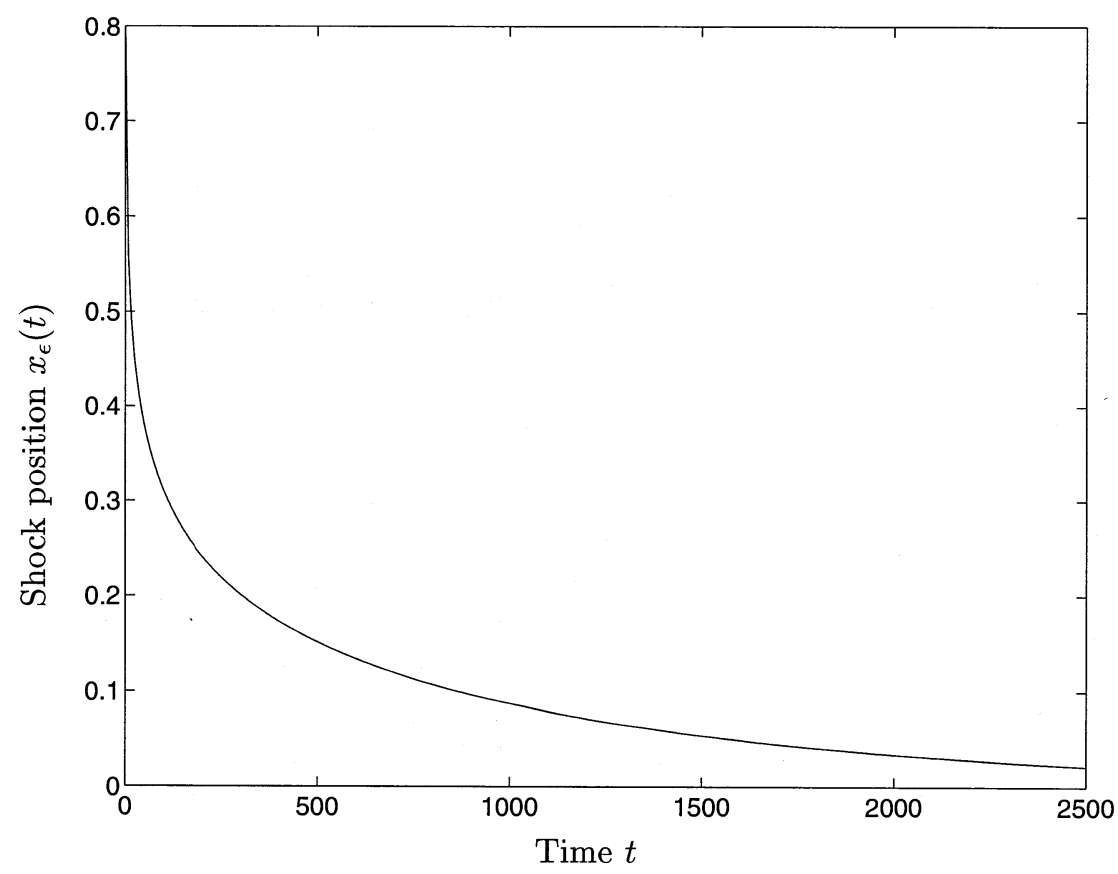

Figure 3. Shock layer motion. 
Figures 2 and 3 illustrate the very slow monotonic motion of the Burgers' shock from $x_{0}=0.8$ to $x_{\epsilon}(\infty)=0$ for the only moderately small $\epsilon=0.1$. Figure 4 shows the same slow shock motion when we instead begin with the continuous initial data

$$
u(x, 0)= \begin{cases}-1, & -1 \leq x \leq 0.6 \\ 5 x-4, & 0.6 \leq x \leq 1\end{cases}
$$

corresponding to $x_{0}=0.8$.

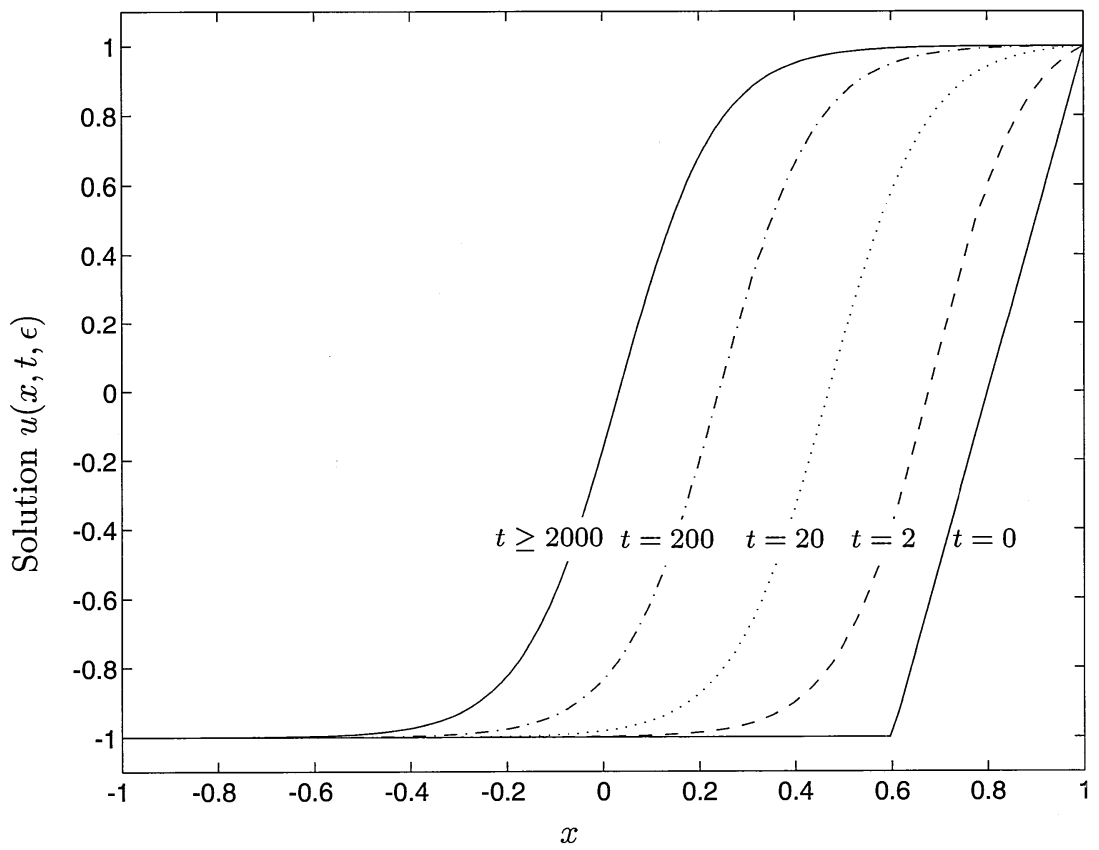

FIgURE 4. Burgers' shock for continuous initial data.

Numerical and analytical work by Allen (1988) and Bohé $(1990,1994)$ shows that the steady-state shock location is extremely sensitive to asymptotically exponentially small changes in the coefficients and boundary data for (1) or (20). Indeed, if we change the right end value in (5) from $u(1, t, \epsilon)=1$ to $1-b e^{-a / \epsilon}$ for some constants $a$ and $b$ with $0<a<1$, the steady-state location $x_{0}(\infty)$ for the limiting Burgers' shock moves from 0 when $b=0$ to $-1+a$ for $b<0$ and to $1-a$ for $b>0$ (cf. Laforgue and O'Malley $(1993,1994)$ and Figures 5 and 6 ). This extreme ill-conditioning and the very slow convergence to the steady state both reflect the existence of an asymptotically exponentially small eigenvalue, $-\frac{2}{\epsilon} e^{-1 / \epsilon}$, for the linearization of the problem about the steady-state (cf. Kreiss (1991) and Laforgue and O'Malley (1994)). Ordinary numerical methods will certainly be challenged to overcome such exponential ill-conditioning, though the WKB-like reformulation and approach of Reyna and Ward (1994a,b) and Ward and Reyna (1994) attain success. 


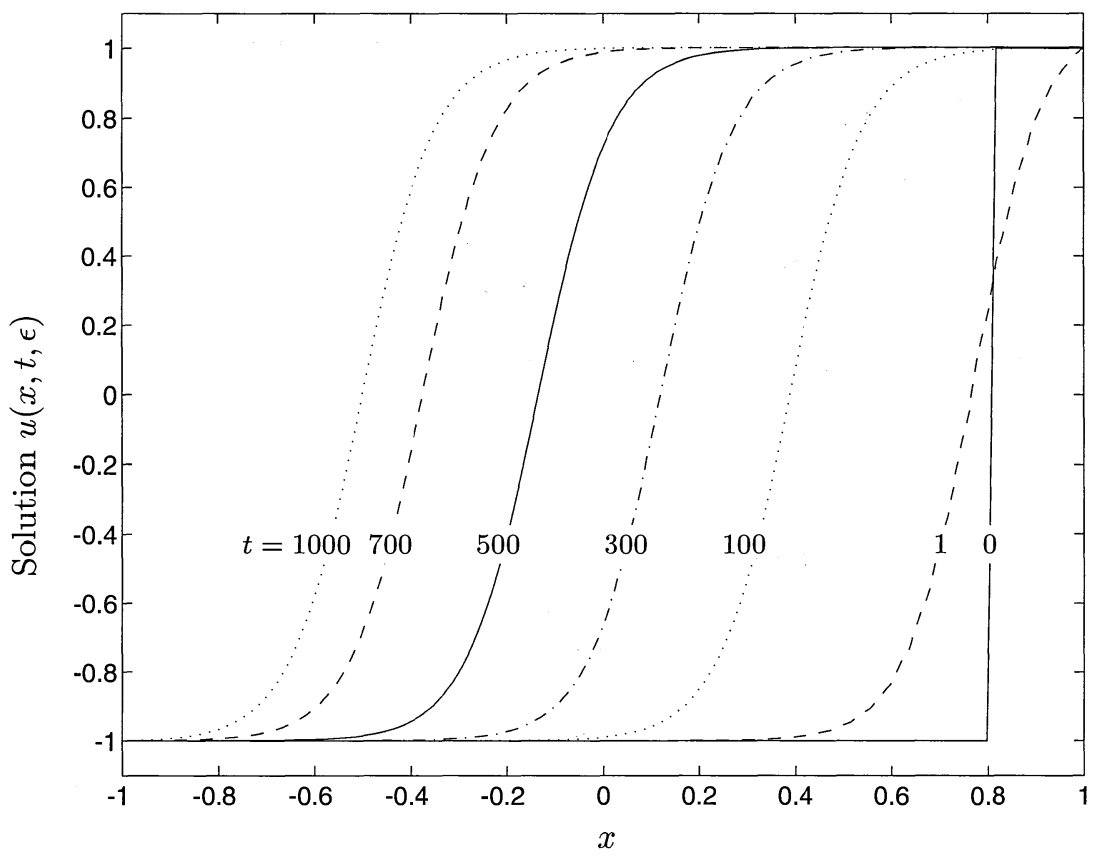

Figure 5. Burgers' shock for $\epsilon=0.075$ and $u(1, t, \epsilon)=1+2 e^{-0.5 / \epsilon}$.

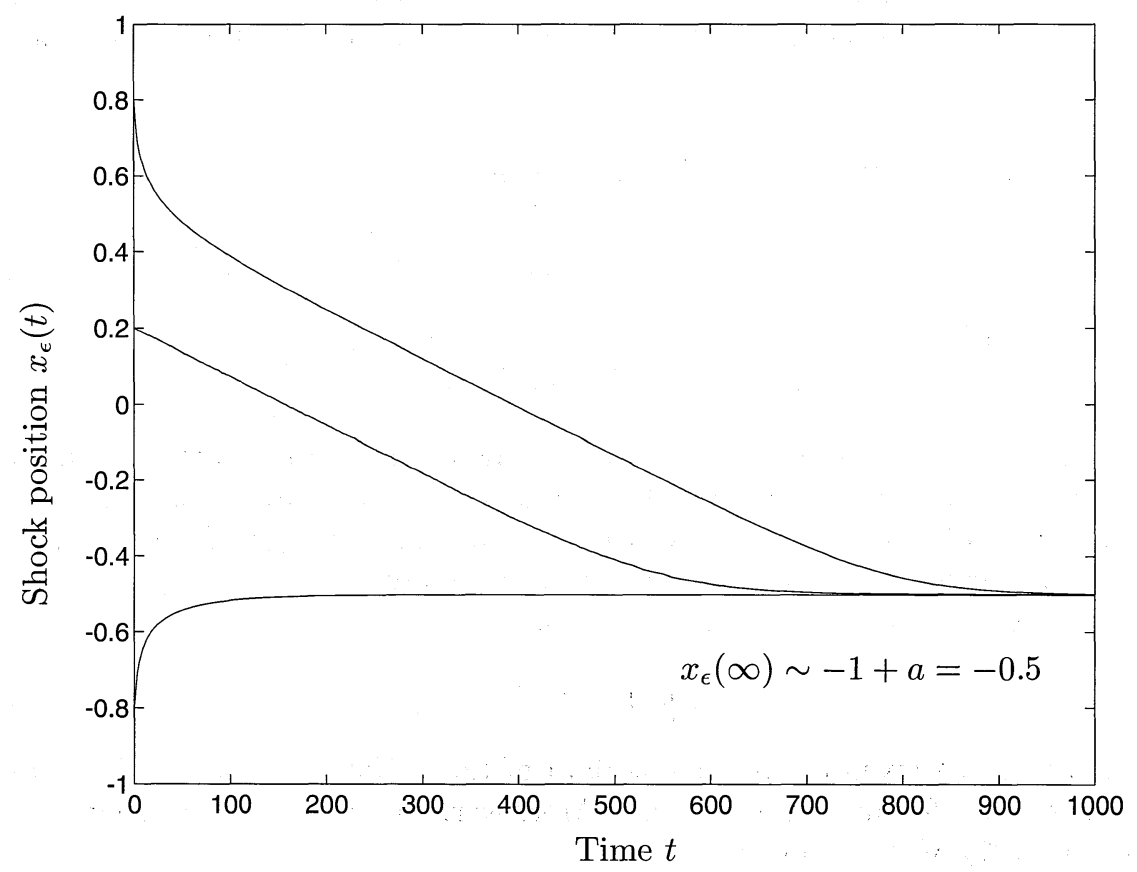

FiguRE 6. Layer motion, starting from $x_{0}=-0.8,0.2$, or 0.8 . 


\section{Long-term shock layer evolution and supersensitivity}

Let us now seek the asymptotic solution $u(x, t, \epsilon)$ of the perturbed problem

$$
\begin{gathered}
\left(\epsilon f(u) u_{x}+G(u)\right)_{x}=u_{t}, \quad-1 \leq x \leq 1, \quad t \geq 0, \\
u(-1, t, \epsilon)=-1, \quad u(1, t, \epsilon)=1-b e^{-a / \epsilon}, \quad a>0, \\
u(x, 0)=\left\{\begin{array}{cc}
-1 & \text { for }-1 \leq x<x_{0} \\
1 & \text { for } x_{0}<x \leq 1 .
\end{array}\right.
\end{gathered}
$$

To determine how the shock now evolves to its steady state, let us introduce the exponentially-compressed time scale

$$
\sigma \equiv t e^{-a / \epsilon}
$$

the corresponding stretched spatial scale

$$
\eta \equiv \frac{x-x_{\epsilon}(\sigma)}{\epsilon}
$$

which is zero at the (slowly-moving) center of the shock, where $u\left(x_{\epsilon}(\sigma), \sigma, \epsilon\right)=0$, and

$$
x_{\epsilon}^{\prime}(0)=x_{0}^{\epsilon} \equiv x_{0}+\epsilon \eta_{0}
$$

with $\eta_{0}$ defined in (17). The scale used in (24) might be motivated by either observing that the exact solution of the perturbed Burgers' equation has the asymptotically exponentially small rate constant $\frac{1}{\epsilon} e^{-a / \epsilon}$ or by conducting numerical experiments. We will show that such a scale, with the substitute $a=A$ defined in (31) below, is fundamental to the shock's evolution in the unperturbed situation when $b=0$. In terms of $\eta$ and $\sigma$, the solution $u(\eta, \sigma, \epsilon)$ of (23) must satisfy the initial-boundary-value problem

$$
\begin{gathered}
\left(f(u) u_{\eta}+G(u)\right)_{\eta}=e^{-a / \epsilon}\left(\epsilon u_{\sigma}-\frac{d x_{\epsilon}}{d \sigma} u_{\eta}\right) \\
\text { on } \eta_{-} \equiv \frac{-1-x_{\epsilon}(\sigma)}{\epsilon} \leq \eta \leq \eta_{+} \equiv \frac{1-x_{\epsilon}(\sigma)}{\epsilon}, \quad \sigma \geq 0 \\
u\left(\eta_{-}, \sigma, \epsilon\right)=-1, u(0, \sigma, \epsilon)=0, \quad u\left(\eta_{+}, \sigma, \epsilon\right)=1-b e^{-a / \epsilon} \\
u(\eta, 0, \epsilon) \text { to be specified. }
\end{gathered}
$$

More carefully than before, (26) is now posed on the algebraically-expanding spatial interval $\left[\eta_{-}, \eta_{+}\right]$, where both $\eta_{ \pm}=O(1 / \epsilon)$ are simultaneously parameterized by $\epsilon$ and $\sigma$. Since the initial $O(\epsilon) t$-interval (on which the shock profile $\varphi(\eta)$ of $(11)$ emerges) becomes asymptotically exponentially small with respect to $\sigma$, we shall neglect it here and simply impose the smoothed initial condition

$$
u(\eta, 0, \epsilon)=\varphi(\eta)
$$

for (26) instead of the given discontinuous data of (23). Note that the initial phase shift $\eta_{0}$ is accounted for in defining $x_{\epsilon}(0)$. Moreover, we shall seek an asymptotic solution to (26)-(27) of the form

$$
u(\eta, \sigma, \epsilon)=\varphi(\eta)+e^{-a / \epsilon} u_{1}(\eta, \sigma, \epsilon)+\cdots,
$$


where we naturally ask that $u_{1}$ satisfy the corresponding linear variational equation

$$
\left(f(\varphi) u_{1}\right)_{\eta \eta}+\left(g(\varphi) u_{1}\right)_{\eta}=-\frac{d x_{\epsilon}}{d \sigma} \varphi_{\eta}
$$

obtained by equating coefficients of $e^{-a / \epsilon}$ in (26). Observe that the time scale selected allows us to capture in the second term of (28) the same exponentially-small size as the given perturbation in the boundary condition. A higher-order approximation than (28) would, most likely, require the use of a multiple-time scale approach (cf. Kevorkian and Cole (1981)). Note that the leading term $\varphi(\eta)$ in (28) is not stationary, since $\eta$ varies slowly with $\sigma$. Moreover, because $\varphi$ fails to satisfy the end conditions of (26) by asymptotically exponentially small amounts (cf. (12) and (13)), and because the exact size of these quantities is not yet known due to their dependence upon $x_{\epsilon}(\sigma)$ through $\eta$, we shall ask the correction term $e^{-a / \epsilon} u_{1}$ to compensate by satisfying the boundary conditions $e^{-a / \epsilon} u_{1}\left(\eta_{-}, \sigma, \epsilon\right)=-L_{-} e^{A_{-} \eta_{-}}$and $e^{-a / \epsilon} u_{1}\left(\eta_{+}, \sigma, \epsilon\right)=-b e^{-a / \epsilon}+$ $L_{+} e^{-A_{+} \eta_{+}}$. We now rewrite these conditions as

$$
\begin{gathered}
u_{1}\left(\eta_{-}, \sigma, \epsilon\right)=-L_{-} e^{(a-A) / \epsilon} e^{\left(\beta_{0}-x_{\epsilon}\right) A_{-} / \epsilon}, \\
u_{1}\left(\eta_{+}, \sigma, \epsilon\right)=-b+L_{+} e^{(a-A) / \epsilon} e^{\left(x_{\epsilon}-\beta_{0}\right) A_{+} / \epsilon},
\end{gathered}
$$

where we have introduced the harmonic mean

$$
A \equiv \frac{2 A_{+} A_{-}}{A_{+}+A_{-}}>0
$$

and the "steady-state limit"

$$
\beta_{0}=\frac{A_{+}-A_{-}}{A_{+}+A_{-}}
$$

both basic to solving the unperturbed problem when $b=0$. We proceed to integrate the translation-invariant equation $(29)$ with the side condition $u_{1}(0, \sigma, \epsilon)=0$. We first obtain

$$
\left(f(\varphi) u_{1}\right)_{\eta}+g(\varphi) u_{1}=-\frac{d x_{\epsilon}}{d \sigma} \varphi+\gamma
$$

for some integration constant $\gamma(\sigma, \epsilon)$. Since $\varphi_{\eta}=(G( \pm 1)-G(\varphi)) / f(\varphi)$ satisfies the homogeneous equation, another integration implies that

$$
u_{1}(\eta, \sigma, \epsilon)=\varphi_{\eta}(\eta) \int_{0}^{\eta} \frac{\gamma-\frac{d x_{\epsilon}}{d \sigma} \varphi(\zeta)}{G( \pm 1)-G(\varphi(\zeta))} d \zeta
$$


Applying the boundary conditions (30) then uniquely determines the two unknowns $\gamma$ and $\frac{d x_{\epsilon}}{d \sigma}$ in (32). Solving for $\frac{d x_{\epsilon}}{d \sigma}$, we find, in particular, the differential equation

$$
\begin{aligned}
\frac{d x_{\epsilon}}{d \sigma} \varphi_{\eta}\left(\eta_{-}\right) \varphi_{\eta}\left(\eta_{+}\right)\left[\int_{0}^{\eta_{+}} \frac{\varphi(\zeta) d \zeta}{G(1)-G(\varphi(\zeta))} \int_{0}^{\eta_{-}} \frac{d \zeta}{G(1)-G(\varphi(\zeta))}\right. \\
\left.\quad-\int_{0}^{\eta_{+}} \frac{d \zeta}{G(1)-G(\varphi(\zeta))} \int_{0}^{\eta_{-}} \frac{\varphi(\zeta) d \zeta}{G(-1)-G(\varphi(\zeta))}\right] \\
=-L_{-} e^{(a-A) / \epsilon} e^{\left(\beta_{0}-x_{\epsilon}\right) A_{-} / \epsilon} \varphi_{\eta}\left(\eta_{+}\right) \int_{0}^{\eta_{+}} \frac{d \zeta}{G(1)-G(\varphi(\zeta))} \\
\quad+\left(b-L_{+} e^{(a-A) / \epsilon} e^{\left(x_{\epsilon}-\beta_{0}\right) A_{+} / \epsilon}\right) \varphi_{\eta}\left(\eta_{-}\right) \int_{0}^{\eta_{-}} \frac{d \zeta}{G(-1)-G(\varphi(\zeta))}
\end{aligned}
$$

for $x_{\epsilon}$. The estimates (12) and (13), moreover, imply that

$$
\varphi_{\eta}\left(\eta_{ \pm}\right) \int_{0}^{\eta_{ \pm}} \frac{(\varphi(\zeta))^{p} d \zeta}{G( \pm 1)-G(\varphi(\zeta))} \sim \frac{( \pm 1)^{p}}{g( \pm 1)}
$$

for $p=0$ and 1 , neglecting only asymptotically exponentially small terms. It therefore follows that the shock layer location $x_{\epsilon}(\sigma)$ can be found by solving the differential equation

$$
\begin{gathered}
(2+\cdots) \frac{d x_{\epsilon}}{d \sigma}=g(1) b(1+\cdots)-e^{(a-A) / \epsilon}\left[g(-1) L_{-} e^{\left(\beta_{0}-x_{\epsilon}\right) A_{-} / \epsilon}(1+\cdots)\right. \\
\left.+g(1) L_{+} e^{\left(x_{\epsilon}-\beta_{0}\right) A_{+} / \epsilon}(1+\cdots)\right]
\end{gathered}
$$

on $\sigma \geq 0$ with the initial value $x_{0}^{\epsilon}$. Here, the ellipses denote asymptotically exponentially small terms.

For the unperturbed problem with $b=0$, we take $a=A$ to obtain

$$
2 e^{A / \epsilon} \frac{d x_{\epsilon}}{d t}=-g(-1) L_{-} e^{\left(\beta_{0}-x_{\epsilon}\right) A_{-} / \epsilon}-g(1) L_{+} e^{\left(x_{\epsilon}-\beta_{0}\right) A_{+} / \epsilon}+\cdots,
$$

corresponding to an analogous differential equation in Reyna and Ward (1994:a). This result for the unperturbed problem is quite basic. The fundamental expansion parameter here is $e^{-A / \epsilon}$, corresponding to the known size of the dominant eigenvalue for Burgers' equation, the more general eigenvalue estimate of Reyna and Ward (1994a), and the physical argument of Elphick et al. (1990), based on assuming weak interactions between the shock and the boundaries. Most significantly, our analysis verifies an improved (viscous) version of the Rankine-Hugoniot condition. This follows from integrating the differential equation (4) over $-1 \leq x \leq 1$ to obtain

$$
\frac{d}{d t} \int_{-1}^{1} u(x, t, \epsilon) d x=\left.\left(\epsilon f(u) u_{x}+G(u)\right)\right|_{-1} ^{1}=\epsilon\left(f(1) u_{x}(1, t, \epsilon)-f(-1) u_{x}(-1, t, \epsilon)\right),
$$

since $G(u(-1))=G(u(1))$. However, $u \sim \varphi\left(\left(x-x_{\epsilon}\right) / \epsilon\right)$ implies that $u \sim-1$ on $-1<$ $x<x_{\epsilon}, u \sim 1$ on $x_{\epsilon}<x<1$, and $\epsilon u_{x}( \pm 1, t, \epsilon) \sim \varphi^{\prime}\left(\left( \pm 1-x_{\epsilon}\right) / \epsilon\right) \sim L_{ \pm} A_{ \pm} e^{-A_{ \pm}\left(1 \mp x_{\epsilon}\right) / \epsilon}$, so we obtain

$$
-2 \frac{d x_{\epsilon}}{d t} \simeq g(1) L_{+} e^{-A_{+}\left(1-x_{\epsilon}\right) / \epsilon}+g(-1) L_{-} e^{-A_{-}\left(1+x_{\epsilon}\right) / \epsilon},
$$


in agreement with (35). It won't be necessary for us to obtain the explicit solution to the initial-value problem. However, in the special case when $A_{+}=A_{-}$, we note that the solution is

$$
x_{\epsilon}(t)=\epsilon\left(\Gamma+\frac{1}{A} \ln \left\{\frac{1+\tanh \left(\frac{A}{2 \epsilon}\left(x_{0}^{\epsilon}-\epsilon \Gamma\right) e^{-t / T}\right.}{1-\tanh \left(\frac{A}{2 \epsilon}\left(x_{0}^{\epsilon}-\epsilon \Gamma\right) e^{-t / T}\right.}\right\}\right)
$$

where

$$
\Gamma=\frac{1}{2 A} \ln \left(\frac{-g(-1) L_{-}}{g(1) L_{+}}\right)
$$

and $T=\frac{\epsilon}{A}\left(-g(-1) g(1) L_{-} L_{+}\right)^{-1 / 2} e^{A / \epsilon}$. In (35), one term on the right will clearly dominate, depending on the position of $x_{\epsilon}$ with respect to $\beta_{0}$, so $x_{\epsilon}(\sigma)$ will move rapidly and monotonically from $x_{0}^{\epsilon}$ to the steady-state location

$$
x_{\epsilon}(\infty)=\beta_{0}+\frac{\epsilon}{A_{+}+A_{-}} \ln \left(\frac{-g(-1) L_{-}}{g(1) L_{+}}\right)+\text {exponentially small terms }
$$

(improving on (22)). Its stability can be further checked by noting that

$$
\frac{\partial}{\partial x_{\epsilon}}\left|\left(\frac{d x_{\epsilon}}{d \sigma}\right)\right|_{x_{\epsilon}(\infty)}<0
$$

For $b \neq 0$ and $a>A$, the second and third terms on the right of (34) dominate, so the asymptotic limit is again that just found for $b=0$.

For $b \neq 0$ and $0<a<A$, we more conveniently rewrite (34) as

$$
\begin{gathered}
2 \frac{d x_{\epsilon}}{d \sigma}=g(1) b(1+\cdots)-g(-1) L_{-} e^{\left(\beta_{-}-x_{\epsilon}\right) A_{-} / \epsilon}(1+\cdots) \\
-g(1) L_{+} e^{\left(x_{\epsilon}-\beta_{+}\right) A_{+} / \epsilon}(1+\cdots)
\end{gathered}
$$

where

$$
-1<\beta_{-}(a) \equiv-1+\frac{a}{A_{-}}<\beta_{0}<\beta_{+}(a) \equiv 1-\frac{a}{A_{+}}<1 .
$$

Note that $\beta_{-}, \beta_{0}$, and $\beta_{+}$coalesce as $a \rightarrow A^{-}$, while $\beta_{ \pm} \rightarrow \pm 1$ as $a \rightarrow 0^{+}$. Now suppose $b<0$. Then, if $-1<x_{0}^{\epsilon}<\beta_{-}$, the middle term in (37) dominates, so $x_{\epsilon}(\sigma)$ increases exponentially rapidly toward its steady state $\beta_{-}+O(\epsilon)$. However, if $\beta_{-}<x_{0}^{\epsilon}<\beta_{+}$, the first term dominates and $x_{\epsilon}(\sigma)$ will decrease linearly to $\beta_{-}$over a finite $\sigma$ interval. Finally, if $\beta_{+}<x_{0}^{\epsilon}<1, x_{\epsilon}(\sigma)$ will decrease exponentially fast to $\beta_{+}$and then linearly to $\beta_{-}$. This evolution can be observed in the computed example shown in Figure 6, where $\beta_{ \pm}= \pm 0.5$. Analogous conclusions could be obtained for $b>0$. A solution $x_{\epsilon}(\sigma)$ of the initial-value problem for (37) could also be obtained numerically, but the qualitative results are already obvious. In particular, up to asymptotically exponentially small terms, the steady-state solution is

$$
x_{\epsilon}(\infty)=\left\{\begin{aligned}
\beta_{-}(a)+\frac{\epsilon}{A_{-}} \ln \left(\frac{g(-1) L_{-}}{g(1) b}\right) & \text { if } b<0 \text { and } 0<a<A, \\
\beta_{0}+\frac{\epsilon}{A_{+}+A_{-}} \ln \left(\frac{-g(-1) L_{-}}{g(1) L_{+}}\right) & \text {if } b=0 \text { or } a>A, \\
\beta_{+}(a)+\frac{\epsilon}{A_{+}} \ln \left(\frac{b}{L_{+}}\right) & \text {if } b>0 \text { and } 0<a<A .
\end{aligned}\right.
$$


Since the steady-state location, for $b \neq 0$ and $a<A$, depends linearly on $-\frac{a}{\epsilon}+$ $\ln |b|$, the logarithm of the perturbation in (23), it is appropriate to call problem (23) supersensitive. Moreover, since the limit is nonuniform near $b=0$, we might introduce a perturbation $\pm e^{-a(\epsilon) / \epsilon}$, letting $a$ vary with $\epsilon$, in order to numerically obtain the spectrum of possible limits within $\left(\beta_{-}, \beta_{+}\right)$. Note that we will obtain an endpoint layer, instead of an interior shock, as $a \rightarrow 0^{+}$. When $a>A$, the perturbation is too insignificant compared to the fundamental small parameter $e^{-A / \epsilon}$, so it no longer shifts the limiting steady state away from $\beta_{0}$. When $b \neq 0$, however, the $O(\epsilon)$ term in (39) is nonuniform near $a=A$. In particular, for $a=A,(34)$ has the steady state $x_{\epsilon}(\infty)=\beta_{0}+\epsilon \ln Y$ where $Y$ is the unique positive root of $g(1) L_{+} Y^{A_{+}+A_{-}}-$ $g(1) b Y^{A_{-}}+g(-1) L_{-}=0$. Finally, observe the breakdown of the steady-state limits (39) as either $g(-1)$ or $g(1)$ tends to zero and the resulting need for more analysis of the asymptotics of the profile $\varphi(\eta)$ of $(11)$.

In the special case of Burgers' equation, we obtain the exact solution (and a justification for our asymptotic formalism) through use of the remarkable Cole-Hopf transformation

$$
v(x, t, \epsilon)=e^{\frac{1}{2 \epsilon} \int_{0}^{x} u(s, t, \epsilon) d s} .
$$

Introducing the stretched variables $\zeta=x / 2 \epsilon$ and $\tau=t / 4 \epsilon, v(\zeta, \tau, \epsilon)$ will satisfy the heat equation. Separating variables, we readily get the Fourier series solution

$$
\begin{aligned}
v(\zeta, \tau, \epsilon)=\alpha_{0} \cosh \left(\nu_{0}\left(\zeta-\gamma_{0}\right)\right) e^{\nu_{0}^{2} \tau}+\beta_{0} \sinh \left(\lambda_{0}\left(\zeta-\delta_{0}\right)\right) e^{\lambda_{0}^{2} \tau} & \\
& +\epsilon \sum_{j=1}^{\infty}\left(\alpha_{j} \cos \left(\nu_{j}\left(\zeta-\gamma_{j}\right)\right) e^{-\nu_{j}^{2} \tau}+\beta_{j} \sin \left(\lambda_{j}\left(\zeta-\delta_{j}\right)\right) e^{-\lambda_{j}^{2} \tau}\right)
\end{aligned}
$$

where all the unknown coefficients can be explicitly determined through the initial and boundary conditions. Long-term behavior is predominantly determined by only the first two terms. For $b=-2$ and $0<a<1$, for example, the boundary conditions imply that

$$
\begin{aligned}
& \nu_{0}=1+2 \epsilon^{-a / \epsilon}+2 e^{-(2-a) / \epsilon}+\cdots, \\
& \lambda_{0}=1-2 e^{-(2-a) / \epsilon}+\cdots
\end{aligned}
$$

and

$$
\gamma_{0}=\frac{1}{2 \epsilon}(-1+a)+\cdots
$$

(cf. Laforgue and O'Malley (1994) and (1995a)). The exponentially small difference between the first two eigenvalues $\nu_{0}$ and $\lambda_{0}$ (for $\epsilon$ small) ultimately becomes significant, as does the phase shift in the leading term. Inverting the Cole-Hopf transformation, we get the solution

$$
\begin{aligned}
u=\frac{v_{\zeta}}{v} & =\nu_{0} \tanh \nu_{0}\left(\frac{x+1-a}{2 \epsilon}+\cdots\right)+O\left(\frac{\beta_{0}}{\alpha_{0}} e^{(1-a) / \epsilon} e^{-\left(t e^{-a / \epsilon}\right) / \epsilon}\right) \\
& \simeq \tanh \left(\frac{x-x_{\epsilon}\left(t e^{-a / \epsilon}\right)}{2 \epsilon}\right) .
\end{aligned}
$$

This confirms the supersensitivity of the perturbation, the slow approach to steady state, and the nearly constant profile. 
It is critical to realize that our success in analyzing the long-term shock layer motion and in describing its supersensitivity is because we carefully accounted for asymptotically exponentially small effects. This reflects the fact that the linearized problem (29)-(30) for the correction term $u_{1}$ has an asymptotically exponentially small eigenvalue. We obtained the differential equation for the shock layer's motion by solving the boundary-value problem for the appropriately scaled correction $u_{1}$ to the shock profile $\varphi$ in the assumed asymptotic solution (28) to our perturbed problem (23). The analyses by Reyna and Ward invoke a projection or orthogonality related to the approximate eigenvector $\varphi_{\eta}$ corresponding to the exponentially small eigenvalue (cf. also Elphick et al. (1990) and Brown et al. (1994)). The need to do such "asymptotics beyond all orders" arises in an increasing number of significant applications (cf. Segur et al. (1991)). Lange (1983) was a significant precursor.

\section{Two more general problems}

(a) Let us first consider the multi-perturbed advection-diffusion problem

$$
\begin{gathered}
{\left[\epsilon f(u) u_{x}+G(u)+b_{0}(u) e^{-a / \epsilon}\right]_{x}=u_{t} \quad \text { on }-1 \leq x \leq 1 \text { and } t \geq 0} \\
u( \pm 1, t, \epsilon) \pm \epsilon c_{ \pm} u_{x}( \pm 1, t, \epsilon)= \pm 1+b_{ \pm} e^{-a / \epsilon} \\
u(x, 0, \epsilon)= \begin{cases}-1, & -1 \leq x<x_{0} \\
1, & x_{0}<x \leq 1\end{cases}
\end{gathered}
$$

where $f, G$, and $b_{0}$ are smooth, $f>0, G=\int_{0}^{u} g(s) d s$ satisfies $(6), 0 \leq c_{ \pm} A_{ \pm}<1$ for $A_{ \pm}= \pm g( \pm 1) / f( \pm 1), a>0$, and $\epsilon \rightarrow 0^{+}$. Compared with (4)-(6), we have introduced three asymptotically exponentially small perturbations of the same order and somewhat more general boundary conditions. Due to the nonnegativity of $c_{ \pm}$, the corresponding shock layer profile $\varphi(\eta)$ will again satisfy $\varphi( \pm \infty)= \pm 1$. Thus, $\varphi$ with $\varphi(0)=0$ will again be defined implicitly by (11) and the decay estimates implied by (12) and (13) for $\varphi(\eta)$ and $\varphi_{\eta}(\eta)$ will also hold as $\eta \rightarrow \pm \infty$. Seeking an asymptotic solution

$$
u(\eta, \sigma, \epsilon)=\varphi(\eta)+e^{-a / \epsilon} u_{1}(\eta, \sigma, \epsilon)+\cdots
$$

for (40) (as in (28)), with $\eta$ (as in (25)) ranging from $\eta_{-}$to $\eta_{+}$(as in (26)), we now require $u_{1}$ to satisfy the linear boundary-value problem

$$
\begin{gathered}
\left(f(\varphi) u_{1}\right)_{\eta \eta}+\left(g(\varphi) u_{1}+b_{0}(\varphi)\right)_{\eta}=-\frac{d x_{\epsilon}}{d \sigma} \varphi_{\eta} \\
u_{1}\left(\eta_{ \pm}, \sigma, \epsilon\right) \pm c_{ \pm} u_{1 \eta}\left(\eta_{ \pm}, \sigma, \epsilon\right)=b_{ \pm} \pm L_{ \pm}\left(1-c_{ \pm} A_{ \pm}\right) e^{a / \epsilon} e^{\mp A_{ \pm} \eta_{ \pm}}
\end{gathered}
$$

The general solution of the linear ordinary differential equation for $u_{1}$ with $u_{1}(0, \sigma, \epsilon)=$ 0 is

$$
u_{1}(\eta, \sigma, \epsilon)=-\varphi_{\eta}(\eta) \int_{0}^{\eta}\left[\frac{b_{0}(\varphi(\zeta))+\frac{d x_{\epsilon}}{d \sigma} \varphi(\zeta)-\gamma}{G( \pm 1)-G(\varphi(\zeta))}\right] d \zeta
$$

for some arbitrary constant of integration $\gamma(\sigma, \epsilon)$. Applying the boundary conditions at $\eta_{ \pm}$will uniquely determine the unknowns $\frac{d x_{\epsilon}}{d \sigma}$ and $\gamma$ in (42). Solving, as in (33), 
we obtain an initial-value problem

$$
\begin{gathered}
2 \frac{d x_{\epsilon}}{d \sigma} \sim B-g(-1) L_{-}\left(1-c_{-} A_{-}\right) e^{\left(a-A_{-}\left(1+x_{\epsilon}\right)\right) / \epsilon} \\
-g(1) L_{+}\left(1-c_{+} A_{+}\right) e^{\left(a-A_{+}\left(1-x_{\epsilon}\right)\right) / \epsilon} \\
x_{\epsilon}(0)=x_{0}^{\epsilon} \equiv x_{0}+\epsilon \eta_{0}
\end{gathered}
$$

for the shock layer location $x_{\epsilon}(\sigma)$ where

$$
B \equiv g(-1) b_{-}+b_{0}(-1)-g(1) b_{+}-b_{0}(1)
$$

is defined through the perturbations $b_{ \pm} e^{-a / \epsilon}$ and $b_{0}( \pm 1) e^{-a / \epsilon}$ in (40). We determine the same ultimate steady-state shock locations $\beta_{-}, \beta_{0}$, and $\beta_{+}$as in (38), but now the limits corresponding to (39) depend on the $\operatorname{sign}$ of $B$, rather than $b$. We also have the sluggish approach to the steady state, as well as supersensitivity to the perturbations defining $B$. Note that the effects of the various perturbations cancel when $E$ is zero. Reyna and Ward (1994a) show that the shock layer is destabilized when our upper bounds on $c_{ \pm}$are exceeded, as one might anticipate from the resulting sign changes in (43).

(b) Now, let us consider the reaction-diffusion problem

$$
\begin{gathered}
\epsilon^{2}\left(f(u) u_{x}\right)_{x}+h(u)+b_{0}(u) e^{-a / \epsilon}=u_{t}, \\
u( \pm 1, t, \epsilon) \pm \epsilon c_{ \pm} u_{x}( \pm 1, t, \epsilon)= \pm 1+b_{ \pm} e^{-a / \epsilon}, \\
u(x, 0, \epsilon)=\left\{\begin{array}{cc}
-1 & \text { for }-1 \leq x<x_{0}, \\
1 & \text { for } x_{0}<x \leq 1,
\end{array}\right.
\end{gathered}
$$

with natural smoothness assumptions on $f, h$, and $b_{0}$, positivity of $f(u)$ and $a$, the condition that

$$
\begin{gathered}
H(u) \equiv \int_{0}^{u} f(s) h(s) d s \text { satisfies } \\
H(1)=H(-1) \text { and } H(u)<H( \pm 1) \text { for }|u|<1, \\
\text { while } h( \pm 1)=0 \text { and } h^{\prime}( \pm 1)<0,
\end{gathered}
$$

and

$$
0 \leq c_{ \pm} A_{ \pm}<1 \quad \text { for } \quad A_{ \pm} \equiv \sqrt{-h^{\prime}( \pm 1) / f( \pm 1)}
$$

Such problems arise in models of phase separation and coarsening (cf., e.g., Carr and Pego (1989), Fusco and Hale (1989), Reyna and Ward (1994c), and Ward (1994)), though often with Neumann, rather than our nearly Dirichlet, boundary conditions. The resulting "metastability" is of substantial physical interest. Related wave motion in mathematical biology is considered in Britton (1986), Murray (1989), and Grindrod (1991), and a variety of applications are included in Fife (1988). Our asymptotic solution, obtained by introducing an appropriate exponentially compressed time scale, is of direct relevance even when the perturbations in (44) are absent. These ideas are further developed in Laforgue and O'Malley (1995b). 
Introducing the stationary stretched spatial coordinate $\eta=\left(x-x_{0}\right) / \epsilon$, we obtain

$$
\begin{gathered}
\left(f(u) u_{\eta}\right)_{\eta}+h(u)=u_{t}-b_{0}(u) e^{-a / \epsilon}, \\
u\left(\eta_{ \pm}, t, \epsilon\right) \pm c_{ \pm} u_{\eta}\left(\eta_{ \pm}, t, \epsilon\right)= \pm 1+b_{ \pm} e^{-a / \epsilon}, \\
u(\eta, 0, \epsilon)=\left\{\begin{array}{cc}
-1 & \text { for } \eta_{-} \leq \eta<0, \\
1 & \text { for } 0<\eta \leq \eta_{+},
\end{array}\right.
\end{gathered}
$$

where $\eta_{ \pm}=\left( \pm 1-x_{0}\right) / \epsilon$. We will assume that the solution of this problem attains a pseudo-steady state $\varphi(\eta)$ (for moderately large $t$ values) which is defined by the boundary-value problem

$$
\begin{gathered}
\left(f(\varphi) \varphi_{\eta}\right)_{\eta}+h(\varphi)=0, \quad-\infty<\eta<\infty \\
\varphi( \pm \infty)= \pm 1, \quad \varphi(0)=0 .
\end{gathered}
$$

An argument guaranteeing the development of such a profile without an initial layer translation, under convenient bistability assumptions but with different initial conditions, is given in Fife and Hsiao (1988). We have neglected the exponentially small perturbations in (46) and recognized that the positive signs of $c_{ \pm}$provide exponential decay for $\varphi$ as $\eta \rightarrow \pm \infty$. Development of a shock profile $\varphi$ is illustrated numerically in Figure 7 for $f \equiv 1$ and $h=2 u\left(1-u^{2}\right)$, where $\varphi(\eta)=\tanh \eta$. Problem (46) can be solved by multiplying the differential equation through by $f(\varphi) \varphi_{\eta}$ and integrating from either $\pm \infty$ to obtain $f(\varphi) \varphi_{\eta}=\sqrt{2(H( \pm 1)-H(\varphi(\eta))}$. Separating variables then defines $\varphi(\eta)$ implicitly as

$$
\eta=\int_{0}^{\varphi} \frac{f(s) d s}{\sqrt{2(H( \pm 1)-H(s))}}
$$

Since the integrand has a simple pole at $s= \pm 1$, it follows that

$$
\varphi(\eta)= \pm 1 \mp L_{ \pm} e^{\mp A_{ \pm} \eta}+O\left(e^{\mp 2 A_{ \pm} \eta}\right) \quad \text { as } \eta \rightarrow \pm \infty
$$

for positive constants

$$
L_{ \pm} \equiv \exp \left[ \pm \int_{0}^{ \pm 1}\left(\frac{A_{ \pm} f(s)}{\sqrt{2(H( \pm 1)-H(s))}}-\frac{1}{1 \mp s}\right) d s\right]
$$

Estimates can also be obtained for derivatives of $\varphi(\eta)$ as $\eta \rightarrow \pm \infty$, which agree with formal differentiation of (48).

To study the exponentially-long time behavior and the shock layer movement for (44), we now introduce the exponentially-compressed time

$$
\rho=t \epsilon e^{-a / \epsilon}
$$

and the corresponding stretched spatial variable

$$
\eta=\left(x-x_{\epsilon}(\rho)\right) / \epsilon
$$




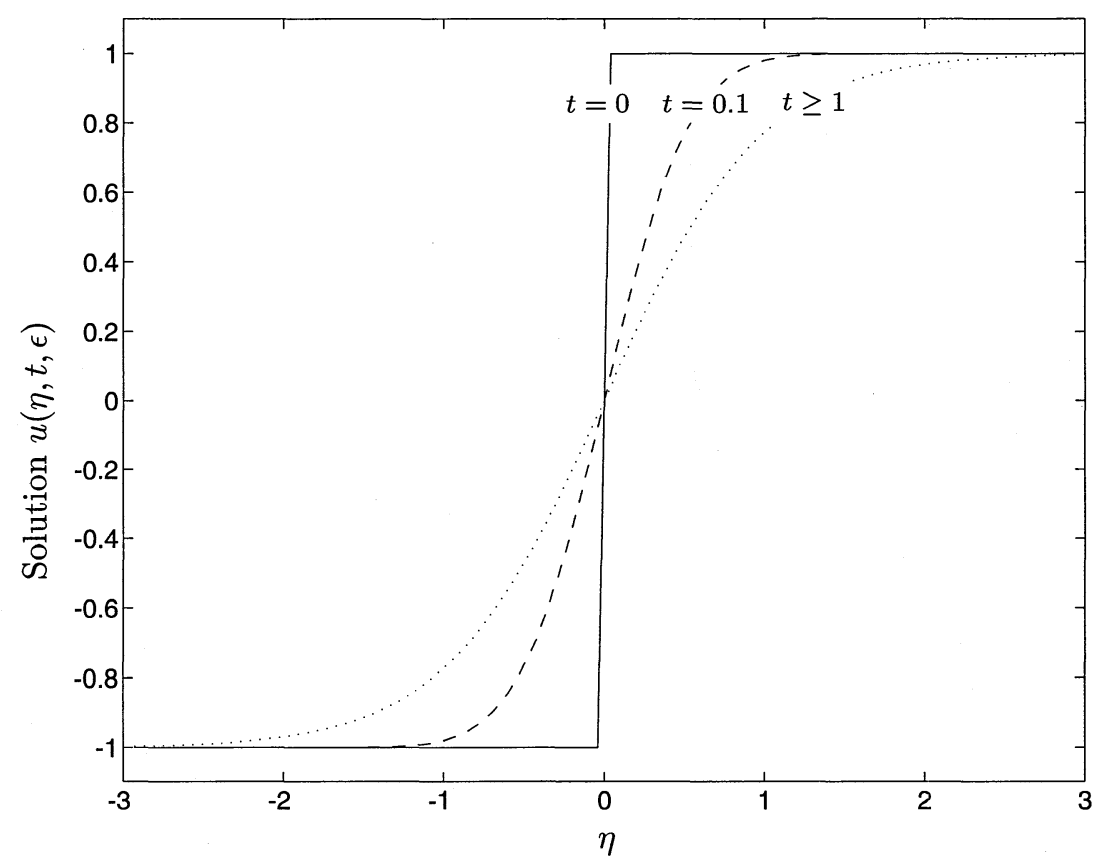

FIgURE 7. Early formation of the profile $\varphi(\eta)$ (shown for $\epsilon=0.1$ ).

centered at the shock layer location $x_{\epsilon}(\rho)$ where the solution $u$ passes through zero. In terms of these new variables, $u(\eta, \rho, \epsilon)$ satisfies

$$
\begin{aligned}
& \qquad\left(f(u) u_{\eta}\right)_{\eta}+h(u)=e^{-a / \epsilon}\left(\epsilon u_{\rho}-\frac{d x_{\epsilon}}{d \rho} u_{\eta}-b_{0}(u)\right) \\
& \text { for } \eta_{-} \equiv-\frac{\left(1+x_{\epsilon}(\rho)\right)}{\epsilon} \leq \eta \leq \eta_{+} \equiv \frac{1-x_{\epsilon}(\rho)}{\epsilon} \quad \text { and } \rho \geq 0 \\
& \qquad \begin{array}{c}
u\left(\eta_{ \pm}, \rho, \epsilon\right) \pm c_{ \pm} u_{\eta}\left(\eta_{ \pm}, \rho, \epsilon\right)= \pm 1+b_{ \pm} e^{-a / \epsilon} \\
\qquad u(\eta, 0, \epsilon)=\varphi(\eta), \quad u(0, \rho, \epsilon)=0
\end{array}
\end{aligned}
$$

Seeking a solution of the form

$$
u(\eta, \rho, \epsilon)=\varphi(\eta)+u_{1}(\eta, \rho, \epsilon) e^{-a / \epsilon}+\cdots,
$$

we naturally ask that $u_{1}$ satisfy the linearized problem

$$
\begin{gathered}
\left(f(\varphi) u_{1}\right)_{\eta \eta}+h^{\prime}(\varphi) u_{1}=-\frac{d x_{\epsilon}}{d \rho} \varphi_{\eta}-b_{0}(\varphi) \\
u_{1}\left(\eta_{ \pm}, \rho, \epsilon\right) \pm c_{ \pm} u_{1 \eta}\left(\eta_{ \pm}, \rho, \epsilon\right)=b_{ \pm} \pm L_{ \pm}\left(1-c_{ \pm} A_{ \pm}\right) e^{\frac{a}{\epsilon}} e^{\mp A_{ \pm} \eta_{ \pm}} \\
u_{1}(0, \rho, \epsilon)=0 .
\end{gathered}
$$

Multiplying this ordinary differential equation in $\eta$ by $f(\varphi) \varphi_{\eta}$ implies that

$$
\begin{aligned}
& {\left[f(\varphi) \varphi_{\eta}\left(f(\varphi) u_{1}\right)_{\eta}-\left(f(\varphi) \varphi_{\eta}\right)_{\eta} f(\varphi) u_{1}\right]_{\eta}} \\
& \quad=-\left[\frac{d x_{\epsilon}}{d \rho} \sqrt{2(H( \pm 1)-H(\varphi))}+b_{0}(\varphi) f(\varphi)\right] \varphi_{\eta}
\end{aligned}
$$


so we have $\left(u_{1} / \varphi_{\eta}\right)_{\eta}$. Integrating, we find that the general solution of (51) with $u_{1}(0, \rho, \epsilon)=0$ is

$$
\begin{aligned}
& u_{1}(\eta, \rho, \epsilon)=-\frac{1}{2} \varphi_{\eta}(\eta) \int_{0}^{\eta} {\left[\int_{0}^{\varphi(\zeta)}\left[\frac{d x_{\epsilon}}{d \rho} \sqrt{2(H( \pm 1)-H(s))}+b_{0}(s) f(s)\right] d s\right.} \\
&+\gamma(\rho, \epsilon)][H( \pm 1)-H(\varphi(\zeta))]^{-1} d \zeta
\end{aligned}
$$

where $\gamma(\rho, \epsilon)$ and $\frac{d x_{\epsilon}}{d \rho}$ both follow from the boundary conditions

$$
\begin{aligned}
-\frac{1}{2}\left(\varphi_{\eta}\left(\eta_{ \pm}\right) \pm c_{ \pm} \varphi_{\eta \eta}\left(\eta_{ \pm}\right)\right) \int_{0}^{\eta_{ \pm}}[\gamma & +\sqrt{2} \frac{d x_{\epsilon}}{d \rho} \int_{0}^{\varphi(\zeta)} \sqrt{H( \pm 1)-H(s)} d s \\
& \left.+\int_{0}^{\varphi(\zeta)} b_{0}(s) f(s) d s\right][H( \pm 1)-H(\varphi(\zeta))]^{-1} d \zeta \\
\mp \frac{c_{ \pm} \varphi_{\eta}\left(\eta_{ \pm}\right)}{2\left(H( \pm 1)-H\left(\varphi\left(\eta_{ \pm}\right)\right)\right.}[\gamma+ & \sqrt{2} \frac{d x_{\epsilon}}{d \rho} \int_{0}^{\varphi\left(\eta_{ \pm}\right)} \sqrt{H( \pm 1)-H(s)} d s \\
& \left.+\int_{0}^{\varphi\left(\eta_{ \pm}\right)} b_{0}(s) f(s) d s\right] \\
= & b_{ \pm} \pm L_{ \pm}\left(1-c_{ \pm} A_{ \pm}\right) e^{\frac{a}{\epsilon}} e^{\mp A_{ \pm} \eta_{ \pm}} .
\end{aligned}
$$

Evaluating the coefficients asymptotically, we obtain

$$
\begin{aligned}
\frac{\gamma e^{ \pm A_{ \pm} \eta_{ \pm}}\left(1+c_{ \pm} A_{ \pm}+\cdots\right)}{2 f( \pm 1) h^{\prime}( \pm 1) L_{ \pm}}+\frac{\sqrt{2}}{2} \frac{d x_{\epsilon}}{d \rho} \frac{\int_{0}^{ \pm 1} \sqrt{H( \pm 1)-H(s)} d s}{f( \pm 1) h^{\prime}( \pm 1) L_{ \pm}} & \times\left(1+c_{ \pm} A_{ \pm}+\cdots\right) e^{ \pm A_{ \pm} \eta_{ \pm}} \\
= \pm b_{ \pm}+L_{ \pm}\left(1-c_{ \pm} A_{ \pm}\right) e^{a / \epsilon} e^{\mp A_{ \pm} \eta_{ \pm}} & \quad\left(\int_{0}^{ \pm 1} b_{0}(s) f(s) d s\right) e^{ \pm A_{ \pm} \eta_{ \pm}} \frac{\left(1+c_{ \pm} A_{ \pm}+\cdots\right)}{2 f( \pm 1) h^{\prime}( \pm 1) L_{ \pm}} \pm \frac{b_{0}( \pm 1)}{h^{\prime}( \pm 1)}+\cdots
\end{aligned}
$$

Finally, eliminating $\gamma$ provides the differential equation for the shock layer location $x_{\epsilon}(\rho)$

$$
\begin{aligned}
\frac{d x_{\epsilon}}{d \rho}\left(\int_{-1}^{1} \sqrt{2(H( \pm 1)-H(s))} d s+\cdots\right) \\
=-\int_{-1}^{1} b_{0}(s) f(s) d s-N_{+} e^{\left(a-2 A_{+}\left(1-x_{\epsilon}\right)\right) / \epsilon}+N_{-} e^{\left(a-2 A_{-}\left(1+x_{\epsilon}\right)\right) / \epsilon} \\
\quad+D_{+} e^{-A_{+}\left(1-x_{\epsilon}\right) / \epsilon}+D_{-} e^{-A_{-}\left(1+x_{\epsilon}\right) / \epsilon}+\cdots
\end{aligned}
$$

where $\eta_{ \pm}$have been rewritten as functions of $x_{\epsilon}(\rho)$ and

$$
\begin{gathered}
N_{ \pm} \equiv-2 f( \pm 1) h^{\prime}( \pm 1) L_{ \pm}^{2}\left(1-c_{ \pm} A_{ \pm}\right) /\left(1+c_{ \pm} A_{ \pm}\right)>0, \\
D_{ \pm} \equiv 2 f( \pm 1) L_{ \pm}\left[b_{ \pm} h^{\prime}( \pm 1)+b_{0}( \pm 1)\right] /\left(1+c_{ \pm} A_{ \pm}\right) .
\end{gathered}
$$

Equation (53) must be integrated over the semi-infinite interval $\rho \geq 0$ with the initial condition $x_{\epsilon}(0)=x_{0}$.

The solution of $(53)$ when $\int_{-1}^{1} b_{0}(s) f(s) d s \neq 0$ is like that of $(37)$ for $b \neq 0$, i.e., the first three terms dominate and the shock layer will move monotonically with increasing 
$\rho$ from $x_{0}$ to one of two different limits for $x_{\epsilon}(\infty)$. Taking the signs of the coefficients in (53) into account, we ultimately attain an asymptotic balance between the first and second (third) terms of the right-hand side when the first term is positive (negative). Thus, we obtain the steady-state limits

$$
x_{\epsilon}(\infty)=\left\{\begin{array}{c}
1-\frac{a}{2 A_{+}}+\frac{\epsilon}{2 A_{+}} \ln \left(-\int_{-1}^{1} b_{0}(s) f(s) d s / N_{+}\right)+\cdots \\
\text { when } \int_{-1}^{1} b_{0}(s) f(s) d s<0 \\
-1+\frac{a}{2 A_{-}}-\frac{\epsilon}{2 A_{-}} \ln \left(\int_{-1}^{1} b_{0}(s) f(s) d s / N_{-}\right)+\cdots \\
\quad \text { when } \int_{-1}^{1} b_{0}(s) f(s) d s>0 .
\end{array}\right.
$$

In these cases, then, the selection of the limiting shock location $\pm\left(1-a / 2 A_{ \pm}\right)$is determined by the perturbation $b_{0}(u) e^{-a / \epsilon}$ in the differential equation and is independent of the perturbations $b_{ \pm} e^{-a / \epsilon}$ in the boundary conditions. The slow evolution of the shock can be analyzed as before. We note that these steady states are ordered so that

$$
-1<\beta_{-}\left(\frac{a}{2}\right) \equiv-1+\frac{a}{2 A_{-}}<\beta_{0} \equiv \frac{A_{+}-A_{-}}{A_{+}+A_{-}}<\beta_{+}\left(\frac{a}{2}\right) \equiv 1-\frac{a}{2 A_{+}}<1
$$

for $0<a<2 A \equiv\left(4 A_{-} A_{+}\right) /\left(A_{+}+A_{-}\right)$. Moreover, the steady states $\beta_{ \pm}$tend to the nearest endpoint as $a \rightarrow 0^{+}$and to $\beta_{0}$ as $a \rightarrow 2 A^{-}$. For $a>2 A$, the second two terms of (53) dominate, as can be seen by rewriting the equation as

$$
\begin{aligned}
& \frac{d x_{\epsilon}}{d \rho}\left(\int_{-1}^{1} \sqrt{2(H( \pm 1)-H(s))} d s+\cdots\right) \\
& \quad=-\int_{-1}^{1} b_{0}(s) f(s) d s+e^{(a-2 A) / \epsilon}\left(-N_{+} e^{2 A_{+}\left(x_{\epsilon}-\beta_{0}\right) / \epsilon}+N_{-} e^{2 A_{-}\left(\beta_{0}-x_{\epsilon}\right) / \epsilon}\right)+\cdots
\end{aligned}
$$

The corresponding steady state

$$
x_{\epsilon}(\infty)=\beta_{0}+\frac{\epsilon}{2\left(A_{+}+A_{-}\right)} \ln \left(N_{-} / N_{+}\right)+\cdots \quad \text { for } a>2 A
$$

is independent of $\int_{-1}^{1} b_{0}(s) f(s) d s$. A separate study for $a=2 A$ will be omitted.

When all the perturbations are absent (i.e., $b_{0}(u)=0$ and $b_{ \pm}=0$ ), any steady state must involve a balance between the second and third terms in (53). Thus, (57) again holds. The steady-state limit is clearly nonuniform for $\int_{-1}^{1} b_{0}(s) f(s) d s$ near zero when $0<a<2 A$. Determining the evolution of the shock for the unperturbed problem is relatively straightforward. Its approach to the steady state will be exponentially slow with respect to time $t$, in contrast to $\rho$. Furthermore, the fundamental expansion parameter $e^{-2 A / \epsilon}$ then represents the asymptotic order of the dominant eigenvalue of the linearized problem. 
When $\int_{-1}^{1} b_{0}(s) f(s) d s=0$, as would occur when $b_{0} f$ is odd, (53) can be rewritten as

$$
\begin{aligned}
e^{A / \epsilon} \frac{d x_{\epsilon}}{d \rho} & \left(\int_{-1}^{1} \sqrt{2(H( \pm 1)-H(s))} d s+\cdots\right) \\
= & D_{-} e^{\left(\beta_{0}-x_{\epsilon}\right) A_{-} / \epsilon}+D_{+} e^{\left(x_{\epsilon}-\beta_{0}\right) A_{+} / \epsilon} \\
& +e^{(a-A) / \epsilon}\left(N_{-} e^{\left(\beta_{0}-x_{\epsilon}\right) 2 A_{-} / \epsilon}-N_{+} e^{\left(x_{\epsilon}-\beta_{0}\right) 2 A_{+} / \epsilon}\right)+\cdots .
\end{aligned}
$$

For $a>A$, the third and fourth terms dominate, so we again obtain (57). For $a<A$, it is essential to realize (cf. (54)) that the signs of $D_{ \pm}$are those of $b_{ \pm} h^{\prime}( \pm 1)+b_{0}( \pm 1)$.

Suppose, first, that $x_{\epsilon}-\beta_{0} \ll-\epsilon$. Then, the first and third terms on the right side of (58) will dominate the second and fourth terms, respectively, presuming $D_{-} \neq 0$. Omitting the negligible terms, we write the remaining equation as

$$
e^{a / \epsilon} \frac{d x_{\epsilon}}{d \rho} \int_{-1}^{1} \sqrt{2(H( \pm 1)-H(s))} d s \sim D_{-} e^{\left(\beta_{-}-x_{\epsilon}\right) A_{-} / \epsilon}+N_{-} e^{2\left(\beta_{-}-x_{\epsilon}\right) A_{-} / \epsilon}+\cdots
$$

where $\beta_{-}(a) \equiv-1+a / A_{-}$as before. Note that $\beta_{-}\left(\frac{a}{2}\right)<\beta_{-}(a)<\beta_{0}$ for $0<a<A$, while $\beta_{-}(0)=-1$ and $\beta_{-}(A)=\beta_{0}$. If $x_{\epsilon}>\beta_{-}$(respectively, $x_{\epsilon}<\beta_{-}$), and $D_{-}<0$, the first (respectively, second) term on the right of the equation dominates, so $x_{\epsilon}(\rho)$ moves monotonically to the stable rest point

$$
x_{\epsilon}(\infty) \simeq-1+\frac{a}{A_{-}}+\frac{\epsilon}{A_{-}} \ln \left(\frac{-N_{-}}{D_{-}}\right),
$$

provided

$$
\int_{-1}^{1} b_{0}(s) f(s) d s=0
$$

and $D_{-}<0$. In particular, then, the limiting steady state will be $\beta_{-}(a)$ if $-1<$ $x_{0}<\beta_{0}$ and $b_{-} h^{\prime}(-1)+b_{0}(-1)<0$. It is supersensitive with respect to both $b_{-}$and $b_{0}(-1)$. This dependence on $x_{0}$ (and a similar dependence observed below) show that the initial condition directly influences the steady state achieved. If $D_{-}>0$, we will instead have $\frac{d x_{\epsilon}}{d \rho}>0$ as long as $x_{\epsilon}-\beta_{0}$ remains $\ll-\epsilon$. Then, any trajectory beginning with $x_{0}<\beta_{0}$ will ultimately reach $\beta_{0}^{-}$.

If we proceed analogously to examine the differential equation for the shock layer when $\beta_{0}<x_{0}<1$, we will find the stable steady state

$$
x_{\epsilon}(\infty) \simeq 1-\frac{a}{A_{+}}+\frac{\epsilon}{A_{+}} \ln \left(D_{+} / N_{+}\right),
$$

provided $b_{+} h^{\prime}(1)+b_{0}(1)>0$. If $D_{+}<0$, we would instead find $x_{0}(\infty) \leq \beta_{0}$.

Finally, we must consider (58) when $\beta_{0}-x_{\epsilon}=O(\epsilon)$. The first two terms on the right of (58) then dominate and we obtain the stable rest point

$$
x_{\epsilon}(\infty) \simeq \beta_{0}+\frac{\epsilon}{A_{+}+A_{-}} \ln \left(-D_{-} / D_{+}\right),
$$

provided $D_{-}>0$ and $D_{+}<0$ (i.e., $\pm\left(b_{ \pm} h^{\prime}( \pm 1)+b_{0}( \pm 1)<0\right)$. With opposite signs, the rest point is unstable. Our preceding analysis then shows that the steady state for (58) will be (59) whenever $x_{0}<\beta_{0}$ and (60) whenever $x_{0}>\beta_{0}$. It also shows that (59) or (60), respectively, hold whenever $D_{+}, D_{-}$, and $\beta_{0}-x_{0}$ are all negative or all 
positive. The cases when either $D_{-}=0$ and $x_{0}>\beta_{0}$ or $D_{+}=0$ and $x_{0}<\beta_{0}$ follow analogously.

Acknowledgements. We are extremely grateful for the insightful comments and suggestions of our friends, Professors Leonid Kalachev, Jerry Kevorkian, and Michael Ward, and of the referees, about this work.

\section{References}

1. J. Allen, unfinished doctoral research, Rensselaer Polytechnic Institute, 1988.

2. N. J. Balmforth, G. R. Ierley, and E. A. Spiegel, Chaotic Pulse Trains, preprint, 1993.

3. H. Bateman, Some recent researches on the motion of fluids, Monthly Weather Review, 43 (1915), 163-170.

4. A. Bohé, Free layers in a singularly perturbed boundary value problem, SIAM J. Math. Anal. 21 (1990), 1264-1280.

5. A. Bohé, Free layers and singular jumps in some singularly perturbed boundary value problems with turning points, Meth. Appl. Anal. 1 (1994), 249-269.

6. N. F. Britton, Reaction-diffusion Equations and their Applications to Biology, Academic Press, London, 1986.

7. D. L. Brown, M. G. Forest, B. J. Miller, and N. A. Petersson, Computation and stability of fluxons in a singularly perturbed sine-Gordon model of the Josephson junction, SIAM J. Appl. Math. 54 (1994), 1048-1066.

8. J. M. Burgers, The Nonlinear Diffusion Equation, Reidel, Dordrecht, 1974.

9. J. Carr and R. L. Pego, Metastable patterns in solutions of $u_{t}=\epsilon^{2} u_{x x}-f(u)$, Comm. Pure Appl. Math. 42 (1989), 523-576.

10. C. Elphick, E. Meron, and E. A. Spiegel, Patterns of propagating pulses, SIAM J. Appl. Math. 50 (1990), 490-503.

11. P. C. Fife, Dynamics of Internal Layers and Diffusive Interfaces, SIAM, Philadelphia, 1988.

12. P. C. Fife and L. Hsiao, The generation and propagation of internal layers, Nonlinear Analysis, 12 (1988), 19-41.

13. C. A. J. Fletcher, Burgers' equation: A model for all reasons, In: Numerical Solutions of Partial Differential Equations, (J. Noye, ed.), North-Holland, Amsterdam, 1982, pp. 139-225.

14. G. Fusco and J. K. Hale, Slow motion manifolds, dormant instability and singular perturbations, J. Dynamics and Differential Equations, 1 (1989), 75-94.

15. M. Garbey, Asymptotic analysis of singular perturbation problems governed by a conservation law, preprint, Argonne National Laboratory, 1989.

16. M. Garbey and J. S. Scroggs, Asymptotic-induced numerical methods for conservation laws, In: Asymptotic Analysis and the Numerical Solution of Partial Differential Equations, (H. G. Kaper and M. Garbey, eds.), Dekker, New York, 1991, pp. 75-96.

17. P. Grindrod, Patterns and Waves, Clarendon Press, Oxford, 1991.

18. F. A. Howes, An analytical treatment of the formation of one-dimensional steady shock waves in uniform and diverging ducts, J. Comp. Appl. Math. 10 (1984), 195-201.

19. —_ Some stability results for advection-diffusion equations, Studies in Applied Math., $\mathbf{7 4}$ (1986), 35-53.

20. A. M. Il'in, Matching of Asymptotic Expansions of Solutions of Boundary Value Problems, Amer. Math. Society, Providence, 1992.

21. A. M. Il'in and O. A. Oleinik, Behavior of the solutions of the Cauchy problem for certain quasilinear equations for unbounded increase of the time, AMS Translations, Series 2, 42 (1964), 19-23.

22. J. Kevorkian, Partial Differential Equations, Wadsworth, Pacific Grove, 1990

23. J. Kevorkian and J. D. Cole, Perturbation Methods in Applied Mathematics, Springer-Verlag, New York, 1981.

24. G. Kreiss, Convergence to steady-state of solutions of viscous conservation laws, In: Asymptotic Analysis and the Numerical Solution of Partial Differential Equations, (H. G. Kaper and M. Garbey, eds.), Dekker, New York, 1991, pp. 225-237.

25. G. Kreiss and H.-O. Kreiss, Convergence to steady state of solutions of Burgers' equation, Appl. Num. Math. 2 (1986), 161-179. 
26. H.-O. Kreiss and J. Lorenz, Initial-Boundary Value Problems and the Navier-Stokes Equations, Academic Press, San Diego, 1989.

27. J. G. L. Laforgue and R. E. O'Malley, Jr., Supersensitive boundary value problems, In: Asymptotic and Numerical Methods for Partial Differential Equations with Critical Parameters, (H. G. Kaper and M. Garbey, eds.), Kluwer, Dordrecht, 1993, pp. 215-223.

28. __ Shock layer movement for Burgers' equation, SIAM J. Appl. Math., 55 (1994).

29. _ Viscous shock motion for advection-diffusion equations, Studies in Appl. Math., 1995a.

30. _ Travelling wave solutions of singularly perturbed reaction-diffusion equations, preprint, $1995 \mathrm{~b}$.

31. C. G. Lange, On spurious solutions of singular perturbation problems, Studies in Appl. Math., 68 (1983), 227-257.

32. P. D. Lax, Hyperbolic Systems of Conservation Laws and the Mathematical Theory of Shock Waves, SIAM, Philadelphia, 1973.

33. J. D. Murray, Singular perturbations of a class of nonlinear hyperbolic and parabolic equations, J. Math. and Physics, 47 (1968), 111-133.

34. __ Mathematical Biology, Springer-Verlag, Heidelberg, 1989.

35. O. A. Oleinik, Discontinuous solutions of non-linear differential equations, Amer. Math. Soc. Translations, series 2, 26 (1963), 95-172.

36. R. E. O'Malley, Jr., Singular perturbations, asymptotic evaluation of integrals, and computational challenges, In: Asymptotic Analysis and the Numerical Solution of Partial Differential Equations, (H. G. Kaper and M. Garbey, eds.), Dekker, New York, 1991, pp. 3-16.

37. L. G. Reyna and M. J. Ward, On the exponentially slow motion of a viscous shock, Comm. Pure Appl. Math., 1994a.

38. _ـ On exponential ill-conditioning and internal layer behavior, J. Num. Func. Anal. Optim., 1994b.

39. __ Resolving weak internal layer interactions for the Cahn-Allen equation, European J. Appl. Math., 1994c.

40. H. Segur, S. Tanveer, and H. Levine, eds., Asymptotics Beyond All Orders, Plenum, New York, 1991.

41. J. Smoller, Shock Waves and Reaction-Diffusion Equations, Springer-Verlag, New York, 1983.

42. M. J. Ward, Metastable patterns, layer collapses, and coarsening for a one-dimensional GinzburgLandau equation, Studies in Appl. Math., 91 (1994), 51-93.

43. M. J. Ward and L. G. Reyna, Internal layers, small eigenvalues, and the sensitivity of metastable motion, SIAM J. Appl. Math, 55 (1994).

44. G. B. Whitham, Linear and Nonlinear Waves, Wiley, New York, 1974.

45. R. Wong, Asymptotic Approximations of Integrals, Academic Press, New York, 1989.

Department of Applied Mathematics, FS-20, University of Washington, Seattle, WashingTON 98195, U.S.A. 